ARTICLE

https://doi.org/10.1038/s41467-020-14333-w

\title{
Manipulating spin polarization of titanium dioxide for efficient photocatalysis
}

\author{
Lun Pan (1) 1,2,7, Minhua Ai ${ }^{1,2,7}$, Chenyu Huang ${ }^{1,7}$, Li Yin ${ }^{3}$, Xiang Liu ${ }^{3}$, Rongrong Zhang ${ }^{1,2}$, Songbo Wang (1) 1,4, \\ Zheng Jiang (1) ${ }^{5,6}$, Xiangwen Zhang ${ }^{1,2}$, Ji-Jun Zou (i) ${ }^{1,2 \star} \&$ Wenbo Mi ${ }^{3 \star}$
}

Photocatalysis has been regarded as a promising strategy for hydrogen production and highvalue-added chemicals synthesis, in which the activity of photocatalyst depends significantly on their electronic structures, however the effect of electron spin polarization has been rarely considered. Here we report a controllable method to manipulate its electron spin polarization by tuning the concentration of $\mathrm{Ti}$ vacancies. The characterizations confirm the emergence of spatial spin polarization among Ti-defected $\mathrm{TiO}_{2}$, which promotes the efficiency of charge separation and surface reaction via the parallel alignment of electron spin orientation. Specifically, $\mathrm{Ti}_{0.936} \mathrm{O}_{2}$, possessing intensive spin polarization, performs 20 -fold increased photocatalytic hydrogen evolution and 8-fold increased phenol photodegradation rates, compared with stoichiometric $\mathrm{TiO}_{2}$. Notably, we further observed the positive effect of external magnetic fields on photocatalytic activity of spin-polarized $\mathrm{TiO}_{2}$, attributed to the enhanced electron-spin parallel alignment. This work may create the opportunity for tailoring the spin-dependent electronic structures in metal oxides.

\footnotetext{
${ }^{1}$ Key Laboratory for Green Chemical Technology of the Ministry of Education, School of Chemical Engineering and Technology, Tianjin University, Tianjin 300072, China. ${ }^{2}$ Collaborative Innovative Center of Chemical Science and Engineering (Tianjin), Tianjin 300072, China. ${ }^{3}$ Tianjin Key Laboratory of Low Dimensional Materials Physics and Preparation Technology, School of Science, Tianjin University, Tianjin 300072, China. ${ }^{4}$ Tianjin Key Laboratory of Brine Chemical Engineering and Resource Eco-utilization, College of Chemical Engineering and Materials Science, Tianjin University of Science \& Technology, Tianjin 300457, China. 5 Shanghai Synchrotron Radiation Facility, Shanghai Institute of Applied Physics, Chinese Academy of Sciences, Shanghai 201204, China. ${ }^{6}$ Shanghai Synchrotron Radiation Facility, Zhangjiang Lab, Shanghai Advanced Research Institute, Chinese Academy of Science, Shanghai 201210,

China. ${ }^{7}$ The authors contributed equally: Lun Pan, Minhua Ai, Chenyu Huang. *email: jj_zou@tju.edu.cn; miwenbo@tju.edu.cn
} 
P hotocatalysis has been regarded as one of the best strategies for hydrogen energy production (from water), environmental remediation (degradation) and synthesis of high value-added chemicals, for which titanium dioxide $\left(\mathrm{TiO}_{2}\right)$ serves as the primary photocatalyst owing to its low cost, inertness, nontoxicity, and strong reducing/oxidizing capabilities ${ }^{1-4}$. The generation of abundant photo-induced electron-hole pairs and their rapid transfer/separation are crucial to maximize the photocatalytic efficiency. To achieve this goal, various strategies have been explored, such as doping with impurity atoms, manipulating exposed facet, introducing vacancies and controlling the morphology and crystal phase ${ }^{1,5-9}$. By these means, the electronic structure of $\mathrm{TiO}_{2}$ is tuned to either extend the light absorption range or accelerate the charge separation ${ }^{1,2,7}$. However, in many cases, the intrinsic mechanism behind these results is still unclear, and especially the spin degree of electronic freedom is rarely considered.

The intrinsic characteristics of electrons, such as electron spin properties, could dominate the property of photocatalyst, so the electronic configuration with different spin states may greatly affect the photocatalytic behaviors. Actually, recent work has shown that the performance of some catalysts can be improved by modulating the spin states ${ }^{10-14}$. For example, the structural distortion in atomically thin nanosheets of $\mathrm{Co}_{3} \mathrm{~S}_{4}, \mathrm{NiSe}_{2}$, and $\mathrm{NiS}$ results in delocalized spin states that provides not only a high electrical conductivity but also a low adsorption energy of reaction intermediates in oxygen evolution reaction (OER) ${ }^{11,15}$. Also, it has been consolidated that the OER kinetics photocatalyzed by $\mathrm{Mn}-\mathrm{N}$ C motifs is dependent on the $e_{\mathrm{g}}$ occupancy of $\mathrm{Mn}^{3+}$ in volcanotype shape with a summit at $c a .0 .95^{16}$. Notably, the induced spin polarization by chiral molecules or chiral films (on $\mathrm{TiO}_{2}, \mathrm{Fe}_{3} \mathrm{O}_{4}$, $\mathrm{CuO}$, etc.) can improve the performances of electrocatalytic and photoelectrochemical (PEC) water splitting ${ }^{17-20}$, by means of suppressing the formation of hydrogen peroxide $\left(\mathrm{H}_{2} \mathrm{O}_{2}\right)$ and favoring the high yield of paramagnetic triplet molecule oxygen (via the parallel spin alignment of oxygen atoms) due to the chiralinduced spin selectivity (CISS) effect ${ }^{21-23}$. Very recently, the external magnetic field has been applied to strengthen the spinrestricted water oxidation process by accelerating the parallel alignment of oxygen radicals during the formation of $\mathrm{O}-\mathrm{O}$ bond ${ }^{24}$. Generally, both electrocatalysis and photocatalysis require rapid charge transfer and long lifetime of intermediated species for redox reactions, so the electron spin property is expected to be an intrinsic factor affecting the performance of photocatalyst.

$\mathrm{TiO}_{2}$ commonly is a nonmagnetic semiconductor due to the lack of unpaired electrons. However, recent researches show that semiconductors like $\mathrm{TiO}_{2}$ and $\mathrm{ZnO}$ with abundant metal vacancies exhibit obvious room-temperature ferromagnetism ${ }^{25-27}$, suggesting the appearance of asymmetric spin-up and spin-down channels in these metal-defected oxides. Also, these photocatalysts show considerably improved activity, but the intrinsic mechanism is still unclear. Fortunately, the above results hint a possible way to modulate the electron spin polarization of metal oxides.

Therefore in this work, we regulate the spin polarization of electrons by controlling the content of metal vacancies and clarify the relationship between the spin polarization and photocatalytic performance. The results imply that the best photocatalytic performance of defected $\mathrm{Ti}_{0.936} \mathrm{O}_{2}\left(\mathrm{TiO}_{2}-10\right)$ is closely related to the high degree of spatial spin polarization, via enhancing the processes of charge separation and surface reaction. Furthermore, we observe the positive effect of the external magnetic field on the photocatalytic performance of spin-polarized $\mathrm{Ti}_{0.936} \mathrm{O}_{2}$. This work may provide a reliable way for tailoring the spin-dependent electronic structures in metal oxides.

\section{Results}

Manipulating metal vacancies in $\mathrm{TiO}_{2}$. Previously, we have found that the encapsulation of $-\mathrm{CH}_{2}-\mathrm{OH}$ groups on $\mathrm{Ti}-\mathrm{O}-\mathrm{Ti}$ skeleton can terminate the crystallization along $a$-axis and lead to the formation of lamellar organometallic compounds (LOC, such as metal glycerolate). Importantly, the thermal oxidation of LOC will remove the surface bonding carbon and hydrogen atoms in the form of $\mathrm{CO}_{2}$ and $\mathrm{H}_{2} \mathrm{O}$, leaving the original oxygen atoms linking with skeleton $\mathrm{Ti}$ atom and causing the oxygen-rich environment, which finally form the inherent Ti defects during the thermal assembly of Ti-O-Ti parallel lattice chains (Supplementary Fig. 1). Accordingly, we expect that the concentration of metal vacancies might be tuned by adjusting the numbers of glycerol groups in glycerolates (the precursor of defected $\mathrm{TiO}_{2}$ ). Therefore, we synthesized a series of glycerolates (G- $m$ ) via solvothermal treatment of tetrabutyl titanate (TBT) with glycerol/ ethanol through changing the content of glycerol ( $m$ refers to the volume of glycerol added, $\mathrm{mL}$ ). During solvothermal synthesis, the glycerol groups replace the butoxy groups of TBT through transesterification reaction. Each glycerol group may share an $\mathrm{O}$ atom to connect two adjacent $\mathrm{Ti}$ atoms to form parallel chain, and accordingly the chain length and layer spacing will change with the number of glycerol groups. XRD analysis (Fig. 1a and Supplementary Figure 2) shows that all glycerolates have the strongest peak at $c a .2 \theta=10.8^{\circ}$ referring to the lamellar peak of metal-based glycerolate ${ }^{25}$. However, the single lamellar peak gradually splits into three peaks with two smaller layer spacings with the increase of $m$. For G-5, the lowest concentration of glycerol groups exists and there are the shortest chain length and largest layer spacing in the unit cell. Then, the chain length becomes longer and the layer spacing becomes smaller with the increase of glycerol dosage from G-5 to G-30, resulting in the better crystallization.

As shown in FT-IR spectra (Fig. 1b and Supplementary Fig. 3), the glycerol C-O stretching vibration bands at 1114 and $1039 \mathrm{~cm}^{-1}$ shift to lower wavenumber from G-5 to G-30, indicating that more glycerol groups coordinate to $\mathrm{Ti}$ cations ${ }^{28,29}$. And Raman spectra (Fig. 1c and Supplementary Fig. 4) show an obvious growth of peak at $723 \mathrm{~cm}^{-1}$ and a decrease of peak at $1077 \mathrm{~cm}^{-1}$, also indicating a structure transition in glycerolates. Importantly, the solid-state CP/ MAS ${ }^{13} \mathrm{C}$ NMR spectra (Fig. 1d) show three well resolved peaks between 70 and $90 \mathrm{ppm}$ (labeled 5) corresponding to the three carbons in glycerolates, in accordance with previous reports ${ }^{28,29}$. Notably, for G-5 there are two small peaks at 20 and $65 \mathrm{ppm}$ (labeled 1 and 3 ) corresponding to the $\left(-\mathrm{CH}_{3},-\mathrm{CH}_{2}-\right)$ and -O$\mathrm{CH}_{2}$ - in butoxy groups ${ }^{30}$, in form of terminal groups due to the insufficient transesterification. With the increase of $m$, the peak at $69 \mathrm{ppm}$ (labeled 4) arises gradually, ascribed to more $-\mathrm{CH}_{2}-\mathrm{O}-$ groups in glycerol connecting to the edge $\mathrm{Ti}$ atom of glycerolates. The small peak at $58 \mathrm{ppm}$ (labeled 2) belongs to the $-\mathrm{CH}_{2}-\mathrm{OH}$ groups, which is only observed for G-5. The specific locations of carbon peaks in ${ }^{13} \mathrm{C}$ NMR spectrum (labeled 1-5) are marked in the molecular structure in Supplementary Fig. 5. The above characterizations confirm the gradually improved crystallization of glycerolates from G-5 to G-30.

The glycerolates $(\mathrm{G}-m)$ were then converted to Ti-defected $\mathrm{TiO}_{2}$ $\left(\mathrm{TiO}_{2}-m\right)$ upon pyrolysis at $470{ }^{\circ} \mathrm{C}$. Thermogravimetric analysis (Supplementary Fig. 6) shows the weight loss gradually increases from G-5 to G-30, due to the increased amount of glycerol bonding with Ti atoms. XRD patterns (Supplementary Fig. 7) and Raman spectra (Supplementary Fig. 8) confirm that all calcined samples can be identified as anatase $\mathrm{TiO}_{2}$ (JCPDS No. 21-1272) without any impurities. What's more, we used standard periclase to calibrate the XRD patterns, and observed a gradual shift of diffraction peaks at $25.31^{\circ}$ to a higher degree (with the $c$ axis being gradually narrowed) from $\mathrm{TiO}_{2}-0$ to $\mathrm{TiO}_{2}-30$ (Fig. $2 \mathrm{a}$, and the 

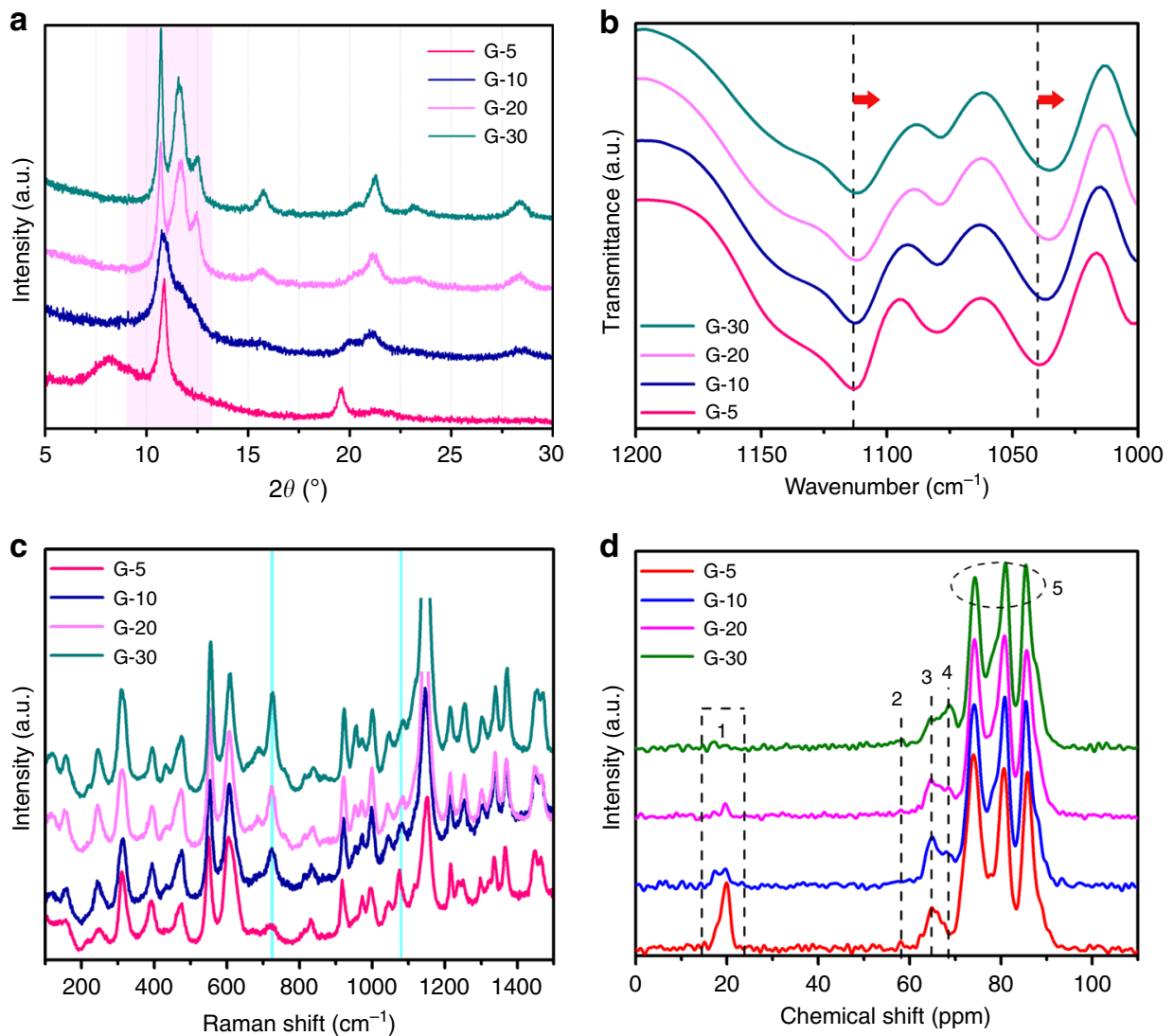

Fig. 1 Crystal structures of as-synthesized titanium glycerolates (G-5, G-10, G-20, and $\mathbf{G - 3 0}$ ). a Enlarged XRD patterns (with $2 \theta$ in the range of $5^{\circ}-30^{\circ}$ ), b Fourier-transform infrared spectroscopy (FT-IR), c Raman spectra, and $\mathbf{d}$ solid-state CP/MAS ${ }^{13} \mathrm{C}$-NMR spectra.

a

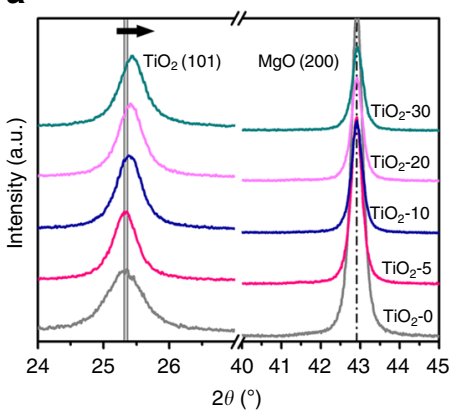

d

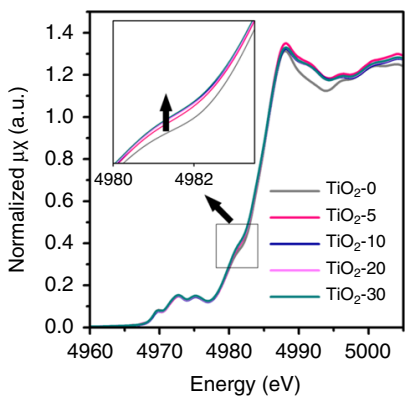

b

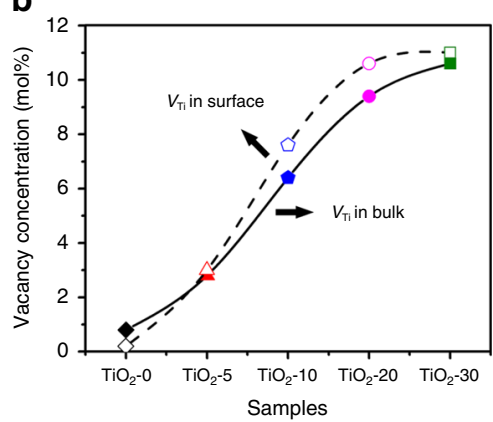

f

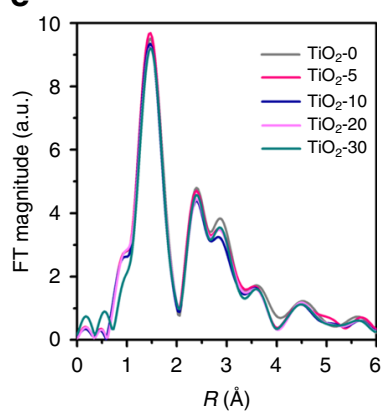

C

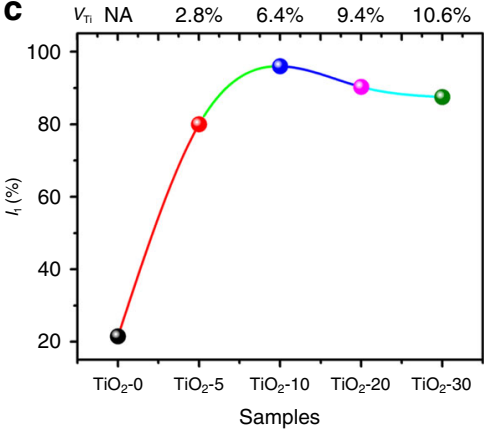

Fig. 2 Defect characterizations of metal-defected $\mathrm{TiO}_{\mathbf{2}}$. a Enlarged XRD patterns (with $2 \theta$ in the range of $24^{\circ}-45^{\circ}$ ); $\mathbf{b} \mathrm{Vacancy}_{\mathrm{concentration} \text { of } \mathrm{TiO}}$ in bulk and surface (determined by chemical titration and XPS analysis, respectively); c Intensity of monovacancies from positron annihilation lifetime spectra (PALS), in which $l_{1}$ is the relative intensity of $\tau_{1}$ (metal monovacancies); $\mathbf{d}$ X-ray absorption near edge structure (XANES) and e Fourier transforms of $k$-space oscillations of Ti K edge; $\mathbf{f}$ high-resolution O1s XPS spectra (fitted); $\mathbf{g}$ The positions of flat-band potential and UV-photoelectron spectroscopy (UPS) VB (valance band) binding energy. 
lattice parameters in Supplementary Table 1), which is consistent with previous report ${ }^{25}$. SEM (Supplementary Fig. 9) and BET (Supplementary Table 2) characterizations indicate all samples possess similar morphology and comparable surface area. In highresolution TEM images (Supplementary Fig. 10), the lattice fringes corresponding to $(101)$ facet $\left(d_{101}=0.35 \mathrm{~nm}\right)$ of anatase $\mathrm{TiO}_{2}$ is clearly observed ${ }^{31}$.

The Ti/O molar ratios in bulk and surface of $\mathrm{TiO}_{2}$ were determined by chemical titration and XPS analysis (the lattice oxygen is fitted from O1s XPS spectra in Supplementary Fig. 11), respectively, and the molar ratio in bulk is very close to that at the surface, indicating the homogenous and uniform composition of defected $\mathrm{TiO}_{2}$. As expected, $\mathrm{TiO}_{2}-0$ contains almost no $\mathrm{Ti}$ vacancies while other samples $(m=5-30)$ possess considerable $\mathrm{Ti}$ vacancies, with $V_{\mathrm{Ti}}$ concentration increasing from $2.8 \%\left(\mathrm{TiO}_{2}-5\right)$ to $10.6 \%\left(\mathrm{TiO}_{2}-30\right)$ (Fig. $2 \mathrm{~b}$ and Supplementary Table 1$)$. The defects in $\mathrm{TiO}_{2}$ were also determined by positron annihilation lifetime spectra (PALS, Supplementary Table 3). The presence of monovacancies can reduce the surrounding electron density and increase the shortest lifetime component of $\tau_{1}{ }^{32,33}$. All defected $\mathrm{TiO}_{2}$ exhibit a longer lifetime $\tau_{1}$ than $\mathrm{TiO}_{2}-0$ ( 264 ps), confirming the existence of a large amount of metal monovacancies. What's more, the relative intensity $\left(I_{1}\right)$ of $\tau_{1}$ provides the relative concentration of metal monovacancies ${ }^{34}$. It can be seen that the concentration of monovacancies increases from $\mathrm{TiO}_{2}-5$ to $\mathrm{TiO}_{2}-10$, then decreases for $\mathrm{TiO}_{2}-20$ and $\mathrm{TiO}_{2}-30$ (Fig. 2c). Correspondingly the intensity of longer lifetime component $\left(I_{2}\right.$ and $\left.I_{3}\right)$ referring to positrons captured by larger size defects (such as dual-, tri-defects) or even large voids ${ }^{32,33}$ are increased for the latter two samples. This result means that $\mathrm{TiO}_{2}-$ 10 has the highest concentration of monovacancies, while some vacancies are in form of vacancy clusters at a high $V_{\mathrm{Ti}}$ concentration $\left(>9 \%, \mathrm{TiO}_{2}-20\right.$, and $\left.\mathrm{TiO}_{2}-30\right)$.

Furthermore, XANES spectra at the Ti K-edge were recorded at room temperature. As shown in Fig. 2d, the defected $\mathrm{TiO}_{2}-m$ samples exhibit similar characteristic lineshape with stoichiometric $\mathrm{TiO}_{2}-0$ (anatase), in both regions of pre-edge peaks $(4964-4980 \mathrm{eV})$ and white line peaks $(4983-5003 \mathrm{eV})$, indicating a high phase purity ${ }^{35}$. However, a gradual intensity increase of the pre-edge peak at $4981 \mathrm{eV}$ from $\mathrm{TiO}_{2}-0$ to $\mathrm{TiO}_{2}-30$ can be observed. Since this peak represents the transition of core electron toward $\mathrm{O} 2 \mathrm{p}$ states hybridizing with the empty Ti4p states ${ }^{35}$, the electron numbers of O2p-Ti4p hybrid orbitals should be decreased gradually from $\mathrm{TiO}_{2}-0$ to $\mathrm{TiO}_{2}-30$, which indicates the presence of lattice $\mathrm{O}$ atoms with unsaturated coordination. Without other impurities, this kind of $\mathrm{O}$ atoms should be caused by the presence of nearby Ti vacancies. Therefore, the intensity of pre-edge peak (at $4981 \mathrm{eV}$ ) is correlated with the $\mathrm{Ti}$ vacancies, which rises gradually from $\mathrm{TiO}_{2}-0$ to $\mathrm{TiO}_{2}-30$.

Meanwhile, the interatomic distances are compared through the Fourier transformed Ti K-edge EXAFS data (Fig. 2e), and all the fitting parameters used by three paths of Ti K-edge EXAFS curves are shown in Supplementary Table 4. A gradual decrease of coordination numbers bonding $\mathrm{Ti}$ atoms for $\mathrm{Ti}_{1}$ is observed from $\mathrm{TiO}_{2}-0$ to $\mathrm{TiO}_{2}-30$ as reflected by the amplitude of $R$ space (Supplementary Table 4), which verifies the appearance of more $\mathrm{Ti}$ vacancies. However, the mean-square disorder of $\mathrm{Ti}_{-} \mathrm{Ti}_{1}$ distance increases first and then decrease, and $\mathrm{TiO}_{2}-10$ owns the highest mean-square disorder, owing to the uniform distribution of monovacancies. The monovacancies will transfer into vacancy clusters in a high defect concentration (such as $\mathrm{TiO}_{2}-30$ ), as confirmed by the decreased mean-square disorder of $\mathrm{Ti}-\mathrm{Ti}$ distance and PALS results.

Additionally, XPS spectra show that the Ti2p peak is symmetrical (Supplementary Fig. 12), without either shoulder peak appearing at $457.9 \mathrm{eV}$ associated with $\mathrm{Ti}^{3+}$ defects or peak at
$457.3 \mathrm{eV}$ from $\mathrm{Ti}-\mathrm{H}$ bonds ${ }^{7,8}$. Moreover, the high-resolution $\mathrm{O} 1 \mathrm{~s}$ spectra (Fig. 2f) reveal the gradual increase of binding energy from $\mathrm{TiO}_{2}-5$ to $\mathrm{TiO}_{2}-30$ because the neighbor $\mathrm{O}$ atoms of $\mathrm{Ti}$ vacancies get less electrons as compared with those on normal sites. Similarly, the DFT calculation shows that the increase of $V_{\mathrm{Ti}}$ content leads to slight increase of oxygen valence (Supplementary Fig. 13). Moreover, the high-resolution C1s spectra (Supplementary Fig. 14) exclude any C-doped impurities.

Besides, all $\mathrm{TiO}_{2}$ samples exhibit similar optical absorption edge at $c a .400 \mathrm{~nm}$, with the band gap of $c a .3 .1 \mathrm{eV}$ (indicated by UV-vis diffuse reflection spectra, Supplementary Figs. 15, 16). Meanwhile, the valence band (VB) (UPS spectra, Fig. $2 \mathrm{~g}$ and Supplementary Fig. 17) shows a gradual shift from $1.45 \mathrm{eV}$ of $\mathrm{TiO}_{2}-5$ to $1.18 \mathrm{eV}$ of $\mathrm{TiO}_{2}-30$ (below the Fermi level), much lower than the case of $\mathrm{TiO}_{2}-0(3.03 \mathrm{eV})$. The results indicate the energy gap between the Fermi level and VB energy level gradually narrows with the increase of $\mathrm{Ti}$ vacancies, which is a $p$-type characteristic ${ }^{36}$. Similarly, a negative slope for defected $\mathrm{TiO}_{2}$ in Mott-Schottky plots (Supplementary Fig. 18) also confirms their p-type conductivity, while $\mathrm{TiO}_{2}-0$ (Supplementary Fig. 19) possessing a normal $n$-type conductivity ${ }^{8,26}$. The position of flat-band potential $\left(E_{\mathrm{FB}} \text {, approximate to the Fermi level }\right)^{37}$ was further calculated, which shifts from $-0.22 \mathrm{~V}\left(\mathrm{TiO}_{2}-0\right)$, to $1.61 \mathrm{~V}\left(\mathrm{TiO}_{2}-5\right)$ and finally to $1.75 \mathrm{~V}\left(\mathrm{TiO}_{2}-30\right)$ vs. NHE (Fig. $2 \mathrm{~g}$ ), similar to UPS VB results. These results confirm the $\mathrm{Ti}$ vacancy is a quadruple shallow acceptor that can push down the Fermi level ${ }^{38}$. Moreover, the carrier density was calculated by means of the Mott-Schottky formula (Supplementary Table 5), and the hole density increases from $\mathrm{TiO}_{2}-5$ to $\mathrm{TiO}_{2}-30$.

Metal-vacancy-dependent spin polarization of $\mathrm{TiO}_{2}$. The above results show that the concentration of metal vacancies in $\mathrm{TiO}_{2}$ can be rationally controlled by adjusting the crystal structure of glycerolate precursors, which provides the possibility to manipulate the electron spin polarization and further the photocatalysis. We applied the Ti L-edge XAS measurements to reveal the complementary electronic properties of $\mathrm{Ti}$ compounds, which reflect the transition from Ti2p orbitals into Ti3d and 4 s orbitals in the conduction band ${ }^{39,40}$. Importantly, the $\mathrm{Ti} \mathrm{L}$ edge spectra contain two sets of peaks, $\mathrm{L}_{3}$ and $\mathrm{L}_{2}$, respectively corresponding to the spin-orbit coupling splitting of initial $2 \mathrm{p}$ states into $2 \mathrm{p}_{3 / 2}\left(\mathrm{~L}_{3}\right)$ and $2 \mathrm{p}_{1 / 2}\left(\mathrm{~L}_{2}\right)^{39}$, which directly refer to the electrons in spin-up (spin quantum number $\left.m_{\mathrm{s}}=+1 / 2\right)$ and spin-down $\left(m_{\mathrm{s}}=-1 / 2\right)$ states, respectively. As shown in Fig. $3 \mathrm{a}, \mathrm{b}$, the $\mathrm{TiO}_{2}-m$ samples show similar $\mathrm{L}_{3}$ peak intensity (spin-up electrons), while the $\mathrm{L}_{2}$ peak intensity (spin-down electrons) first increases from $\mathrm{TiO}_{2}-5$ to $\mathrm{TiO}_{2}-10$, and decreases gradually for $\mathrm{TiO}_{2}-20$ and $\mathrm{TiO}_{2}-30$. The results indicate the increase of $\mathrm{Ti}$ vacancies can raise the amount of electrons in spin-down state (or electron polarization), but too much $\mathrm{Ti}$ vacancies (like $\mathrm{TiO}_{2}-20$ and $\mathrm{TiO}_{2}-30$ ) lead to gradual decrease of electron polarization, may owing to the formation of Ti-vacancies clusters. Notably, the electron polarization of the samples decreases in the order of $\mathrm{TiO}_{2}-10>\mathrm{TiO}_{2}-20>$ $\mathrm{TiO}_{2}-30>\mathrm{TiO}_{2}-5$.

In addition, we calculated the spin polarization properties of Ti-defected $\mathrm{Ti}_{15} \mathrm{O}_{32}$, since its defect concentration is similar to $\mathrm{TiO}_{2}-10$. As shown in Fig. 3c, the DOS of electron spin-up state is much lower than that of spin-down state around the Fermi level, leading to obvious electron polarization that can provide more spin-down photoinduced electrons under light irradiation. As shown in 3D spatial distributions of spin polarization (Fig. 3d), $\mathrm{Ti}_{15} \mathrm{O}_{32}$ exhibits a large area of negative spatial spin polarization (SSP) in the supercell, which indicates a small possibility of spin polarization reversal in the real space ${ }^{41}$. However, for the defected $\mathrm{Ti}_{35} \mathrm{O}_{72}$ with lower $\mathrm{Ti}$ vacancies $(2.78 \%)$, it shows much lower 

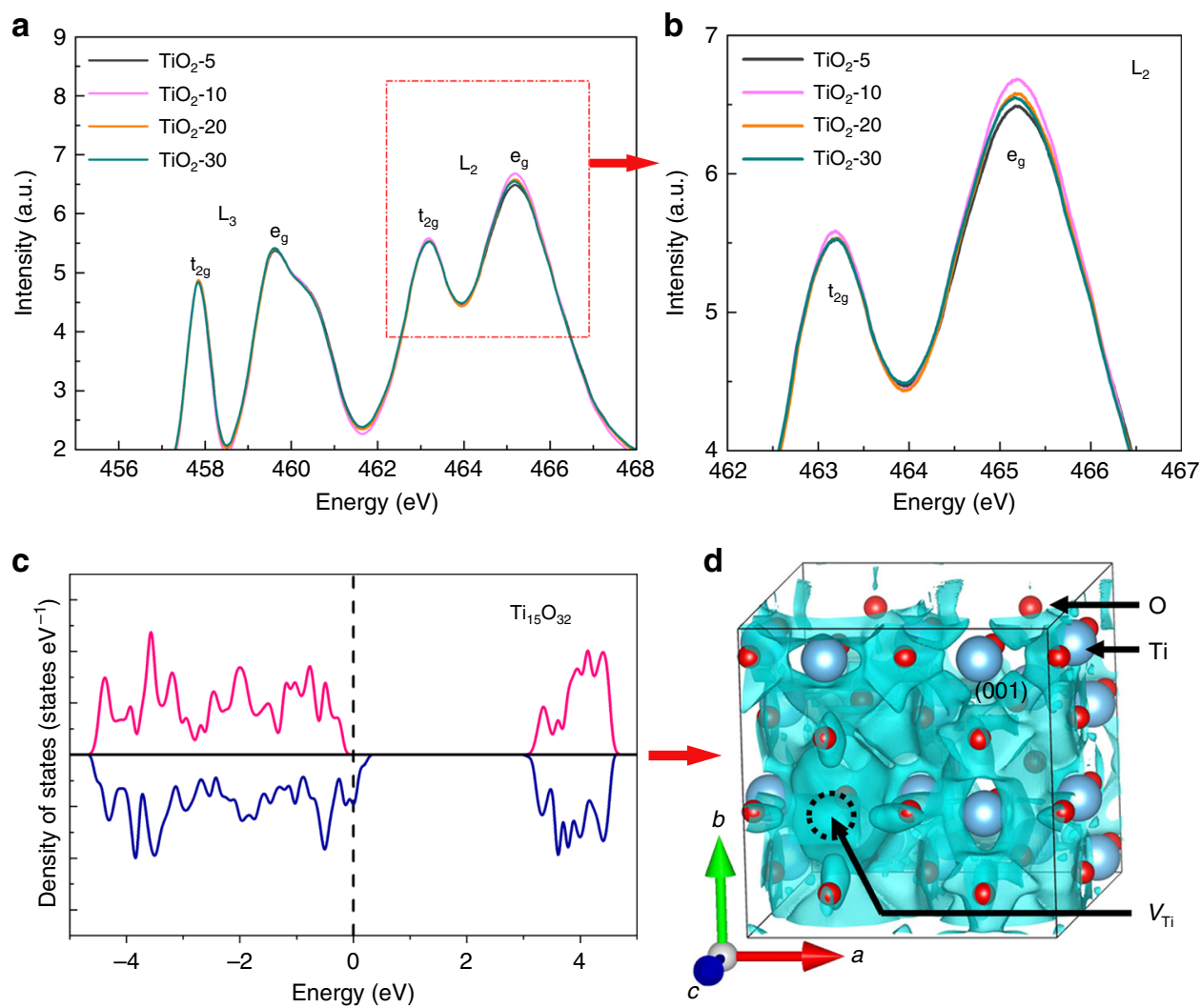

Fig. 3 Spin-dependent electronic structures of metal-defected $\mathrm{TiO}_{\mathbf{2}} \cdot \mathbf{a}, \mathbf{b}$ Ti L-edge and high-resolution $\mathrm{L}_{2} \mathrm{XAS}$ spectra. $\mathbf{c}$ Calculated total density of states (DOS) and $\mathbf{d}$ (001)-Planar 3D spatial distributions of spin polarization (with the energy interval of $\left[E_{F}-0.2 \mathrm{eV}, E_{F}\right]$ ) of metal-defected Ti ${ }_{15} \mathrm{O}_{32}$ model $(6.25 \%$ $V_{T_{\mathrm{i}}}$ ). The specific iso-surface SSP value for $\mathbf{d}$ is $-95 \%$.

spin polarization than $\mathrm{Ti}_{15} \mathrm{O}_{32}$ at the Fermi level (Supplementary Fig. 20), which is consistent with the Ti L-edge XAS spectra. The above results verify the existence of metal-vacancy-dependent spin polarization, and the parallel alignment of electron spin polarization may further promote the efficiencies of charge separation and surface reaction for efficient photocatalysis.

Spin-polarization-dependent photocatalysis. We evaluated the photocatalytic activity of all defected $\mathrm{TiO}_{2}$ samples in hydrogen generation and pollutant degradation under simulated solar light irradiation. As shown in Fig. 4a, the defected samples show much higher $\mathrm{H}_{2}$ generation rate and quantum efficiency $(\mathrm{QE})$ than normal $\mathrm{TiO}_{2}-0$. Notably, the order of activity is $\mathrm{TiO}_{2}-10>\mathrm{TiO}_{2}-$ $20>\mathrm{TiO}_{2}-30>\mathrm{TiO}_{2}-5>\mathrm{TiO}_{2}-0$, and especially $\mathrm{TiO}_{2}-10$ exhibits a 20-fold higher $\mathrm{H}_{2}$ generation rate and 18-fold higher QE than $\mathrm{TiO}_{2}-0$. Moreover, $\mathrm{TiO}_{2}-10$ exhibits high stability without obvious loss in photoactivity after circling experiments (Supplementary Fig. 21). Further for the photodegradation of several organic pollutants, the first-order rate constants $(k)$ of all samples are determined and shown in Fig. 4b and Supplementary Figs. 22-25. As expected, the photodegradation rates also show the same activity order as that of the hydrogen generation. Importantly, $\mathrm{TiO}_{2}-10$ owns the highest photoactivity, whose photoreaction rate (degradation of phenol) is 8 -fold higher than $\mathrm{TiO}_{2}-0$.

Besides, $\mathrm{TiO}_{2}-m$ (with $m=5-30$ ) with p-type conductivity show high cathodic photocurrent in photoelectrochemical (PEC) water splitting and the activity trend is the same with photocatalysis (Fig. 4c), while n-type $\mathrm{TiO}_{2}-0$ can be only applied as photoanode. For n-type $\mathrm{TiO}_{2}-0$, the Fermi level $\left(E_{\mathrm{F}}\right)$ is higher than electrolyte redox level, leading to electrons transfer from
$\mathrm{TiO}_{2}$ to electrolyte with upward band bending, while the $E_{\mathrm{F}}$ of ptype $\mathrm{TiO}_{2}\left(\right.$ like $\left.\mathrm{TiO}_{2}-10\right)$ is lower than electrolyte redox level, resulting in electrons transfer from electrolyte to $\mathrm{TiO}_{2}$ with downward band bending ${ }^{42}$. Accordingly, n-type $\mathrm{TiO}_{2}-0$, with upward band bending at the interface and abundant free electrons, works as the photoanode, while p-type $\mathrm{TiO}_{2}$ (like $\mathrm{TiO}_{2}-10$ ), with downward band bending and abundant free holes, can work as photocathode. Moreover, in electrochemical impedance spectroscopy (EIS) under light irradiation (Supplementary Fig. 26), the radius gradually becomes smaller with the increase of Ti vacancies, suggesting that more Ti vacancies (with the majority carrier of holes) will enhance the electrical conductivity, which agrees with the Mott-Schottky results (Supplementary Table 5).

As described above, the photocatalytic activity of defected $\mathrm{TiO}_{2}$ is in the order of $\mathrm{TiO}_{2}-10>\mathrm{TiO}_{2}-20>\mathrm{TiO}_{2}-30>\mathrm{TiO}_{2}-5>\mathrm{TiO}_{2}-$ 0 , which is the same as that of their electron spin polarizations (Fig. 3). Specifically, with the highest spatial spin polarization, $\mathrm{TiO}_{2}-10$ shows the best photoactivity. The reason for the positive effect of spin polarization on photocatalysis should be dependent on two aspects: the charges separation and surface reaction.

First, the highly spin-polarized electrons can reduce the recombination of photoinduced electrons and holes during the charge transfer process. When a spin-down electron is excited (to $\mathrm{CB}$ ), the remaining hole (in VB) also exhibits the same spin-down characteristic and keeps this spin direction unchanged. During the electron transfer, the original spin direction of electrons will lose (and change to spin-up state) due to spin-orbital coupling, hyperfine interaction, etc ${ }^{43}$. Consequently, the recombination will be inhibited because of the lack of spin-up holes under the environment of high spatial spin polarization, which is similar to the giant magnetoresistance effect ${ }^{44,45}$. Therefore, $\mathrm{TiO}_{2}$ 

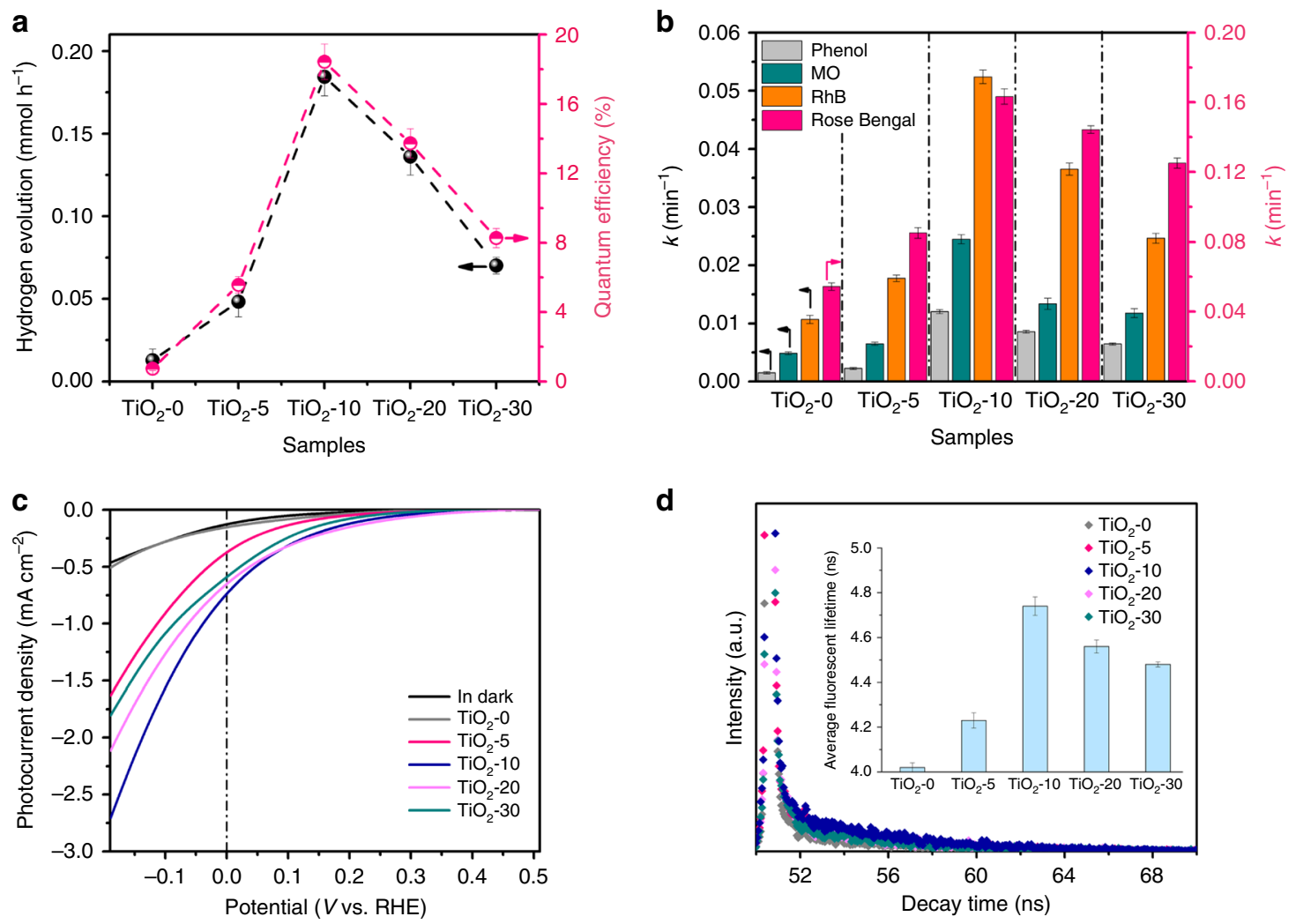

d
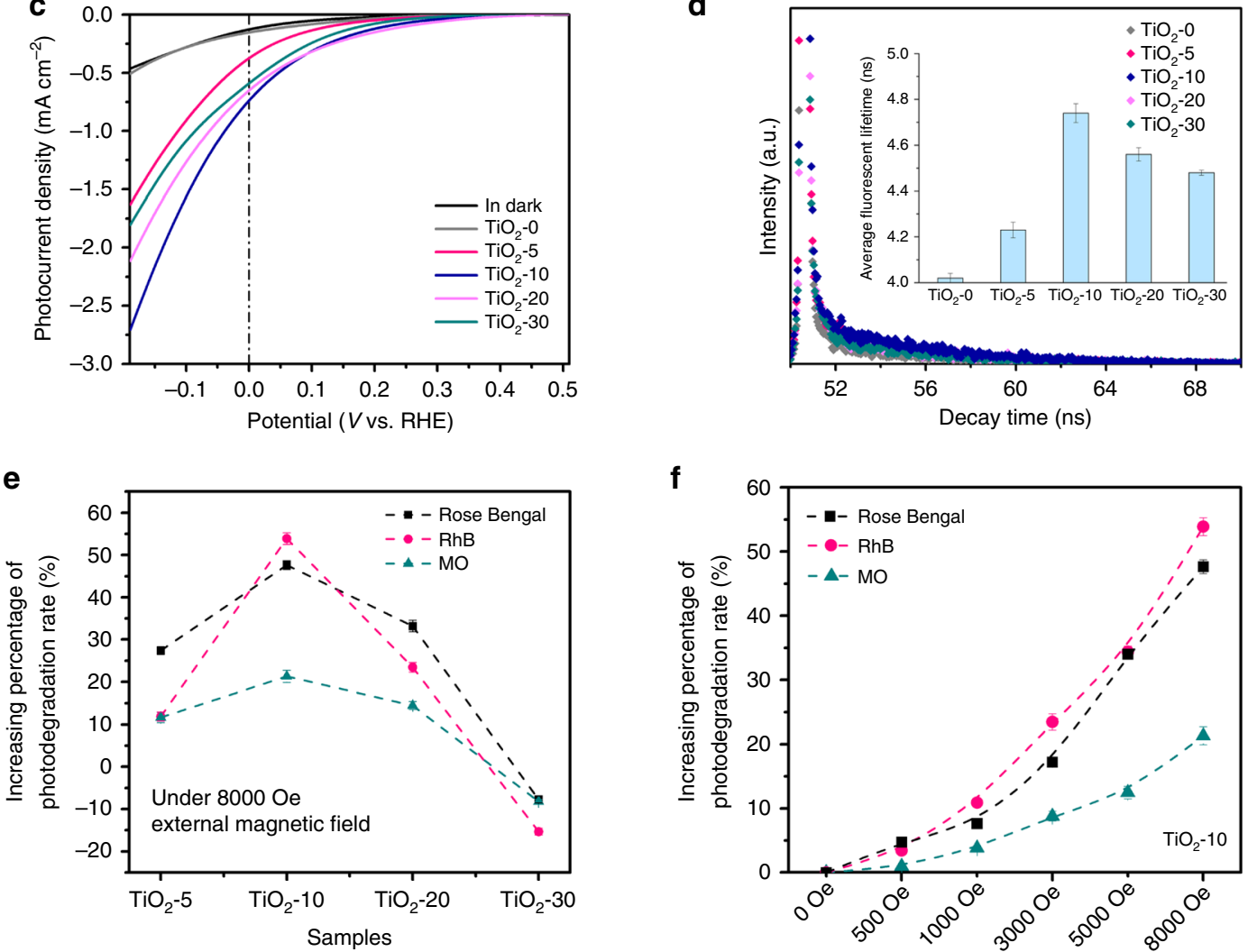

Fig. 4 Photocatalytic performance of metal-defected $\mathbf{T i O}_{\mathbf{2}}$. a Photocatalytic $\mathrm{H}_{2}$ evolution and quantum efficiency (QE); $\mathbf{b}$ The degradation rates of four organic pollutants (Phenol, MO, RhB, Rose Bengal); c Liner sweep voltammetry (LSV) curves of $\mathrm{TiO}_{2}-m$ (photoelectrodes) under light irradiation and in dark; d Time-resolved transient PL decay; e Increasing percentage of photodegradation rates of Ti-defected samples under 8000 Oe external magnetic field; $\mathbf{f}$ Increasing percentage of photodegradation rates of $\mathrm{TiO}_{2}-10$ under different strength of magnetic field from 0 to 8000 Oe.

at $V_{\mathrm{Ti}}=6.4 \%$, with a highest spin polarization (Fig. 3), can inhibit the recombination of photoinduced carriers to the greatest extent. However, for the stoichiometric $\mathrm{TiO}_{2}$ without spin polarization, the excited electron and holes have random spin orientations, and the possibility for one excited electron to recombine with one hole with the same spin orientation is high. As confirmed in the timeresolved transient photoluminescence decay (Fig. 4d and Supplementary Table 6), not surprisingly, $\mathrm{TiO}_{2}-10$ possesses the longest fluorescent lifetime, while other samples show relatively short fluorescent lifetime. Especially, the order of fluorescent lifetime changes in the same order of spin polarization, i.e. $\mathrm{TiO}_{2}-10>$ $\mathrm{TiO}_{2}-20>\mathrm{TiO}_{2}-30>\mathrm{TiO}_{2}-5$.

Meanwhile for the surface reaction, the spin-polarized electrons play an important role in inhibiting the recombination of active species (free radical) such as hydroxyl radicals. Oxygen- containing species like hydroxyl radicals $(\cdot \mathrm{OH})$ are important active species for photocatalysis, which are formed by donating an electron to the hole of $\mathrm{TiO}_{2}$. As discussed above, the spin direction of holes is correlated to the spatial spin polarization, i.e., higher spatial spin polarization leads the higher alignment of hole spin polarization. Therefore, for $\mathrm{TiO}_{2}-10$ with wide spatial spin polarization, the spin-polarized holes (note that the SSP at surface is the same as that in the bulk, Supplementary Fig. 27) can only accept the electrons of $\mathrm{OH}^{-}$(from aqueous solution) with the same spin polarization direction (e.g., spin down), leaving the parallel spin alignment among the $\cdot \mathrm{OH}$ species (e.g., spin up). When the single electrons in different $\cdot \mathrm{OH}$ species are in different spin directions, the hydroxyl radical will combine easily to generate $\mathrm{H}_{2} \mathrm{O}_{2}{ }^{17,22}$, which will reduce the effective proportion of . OHs to take part in the surface oxidation reaction. On the 
contrary, $\cdot$ OHs with single electrons in the same spin direction are not easy to combine ${ }^{17,23,46}$, thus promoting the surface reactions of water splitting and degradation (especially for the case of $\mathrm{TiO}_{2}-10$ ).

It is worth noting that, the above spin-restricted merits can be further enhanced by external magnetic field ${ }^{24}$. Therefore, a magnetic field (easily by the electromagnet) was applied to investigate its effect on the photocatalytic activity of defected $\mathrm{TiO}_{2}$. From Fig. 4e and Supplementary Fig. 28a-c, it can be seen that the magnetic field does enhance the activity of $\mathrm{TiO}_{2}-5, \mathrm{TiO}_{2}-$ 10 , and $\mathrm{TiO}_{2}-20$, which may be attributed to the inhibition of the combination between $\cdot \mathrm{OH}$ species with anti-parallel spin directions ${ }^{17}$. And the effect of magnetic field is most significant for $\mathrm{TiO}_{2}-10$, with the reaction constant increased by $54 \%(\mathrm{RhB}$ degradation) at a magnetic field of $8000 \mathrm{Oe}$, which is related to the its best magnetic feature $\left(0.2 \mu_{\mathrm{B}} /\right.$ unit cell or $3.2 \mu_{\mathrm{B}} /$ supercell, Supplementary Figs. 29-31, similar to the previous result ${ }^{47}$ ) as well as the wideset distribution of spin-polarized electrons ${ }^{24}$. Moreover, for $\mathrm{TiO}_{2}-10$, the degree of photocatalytic enhancement increases with the strength of magnetic field (Fig. 4f and Supplementary Fig. $28 \mathrm{~d}-\mathrm{f}$ ), further confirming the significant positive effect by magnetic field. However, the photocatalytic activity of $\mathrm{TiO}_{2}-30$ is suppressed at an external magnetic field, because the spatial spin polarization reversal (caused by the abundant defects clusters) may appear on the surface, which facilitates the combination of $\cdot \mathrm{OH}$ species.

\section{Discussion}

In summary, we regulated the spin polarization in $\mathrm{TiO}_{2}$ by modulating the content of metal vacancies and illustrated that the increase of spatial spin polarization shows positive effect on the photocatalysis. Especially, the introduced $c a$. $6.4 \% \mathrm{Ti}$ vacancies $\left(\mathrm{TiO}_{2}-10\right)$ leads to intensive spatial spin polarization with wide distribution, which promotes the efficiencies of charge separation and surface reaction via the parallel alignment of electron spin polarizations, and results in significantly promoted photocatalytic activities. Meanwhile, the positive effect of external magnetic fields on Ti-defected $\mathrm{TiO}_{2}$ is also observed, attributed to the enhanced electron-spin parallel alignment. This reliable spinpolarization-modulation strategy via defects tuning gives us inspiration to tailor the spin-dependent electronic structures for highly effective catalysts.

\section{Methods}

Density functional theory calculations. The density functional theory (DFT) calculations were implemented in Vienna Ab initio Simulation Package ${ }^{48}$. DFT with generalized gradient approximation (GGA) provides accurate lattice constants in anatase $\mathrm{TiO}_{2}$, however, the band gap of anatase $\mathrm{TiO}_{2}$ is underestimated by $\mathrm{GGA}^{25}$. In order to improve the band gap, the effective Hubbard $U$ of $7.2 \mathrm{eV}$ is applied for the Ti $3 d$ states $^{49}$ (see the influence of $U$ values on the spatial spin polarization results in Supplementary Fig. 32). Based on the GGA + U method, the calculated band gap of anatase $\mathrm{TiO}_{2}$ is $3.3 \mathrm{eV}$, which is very close to the experimental value of $3.2 \mathrm{eV}$. The supercell size and atomic numbers of normal and 6.25\% Ti-defected $\mathrm{TiO}_{2}$ are listed in Supplementary Table 7.

The energy cutoff for plane wave basis set is $400 \mathrm{eV}$. The convergence criteria for the energy and atomic forces are $10^{-5} \mathrm{eV}$ and $0.01 \mathrm{eV} \AA^{-1}$, respectively. The Brillouin Zone is sampled with $\Gamma$-centered $3 \times 3 \times 3 k$ point meshes for $\mathrm{TiO}_{2}$. The spatial spin polarization (SSP) is defined as:

$$
P(r, z, \varepsilon)=\frac{n_{s}^{\uparrow}(r, z, \varepsilon)-n_{s}^{\downarrow}(r, z, \varepsilon)}{n_{s}^{\uparrow}(r, z, \varepsilon)+n_{s}^{\downarrow}(r, z, \varepsilon)}
$$

where the $n_{s}^{\uparrow(\downarrow)}(r, z, \varepsilon)$ is the spin-up (down) charge density in the real space with an energy interval of $\left[\varepsilon, E_{\mathrm{F}}\right]^{41}$. In this work, the energy range of $\left[E_{F^{-}}-0.2 \mathrm{eV}, E_{F}\right]$ is used (see the effect of energy ranges on SSP results in Supplementary Fig. 33)

Synthesis of $\mathrm{TiO}_{2}$ with metal vacancies. Tetrabutyl titanate $\left[\mathrm{Ti}\left(\mathrm{OC}_{4} \mathrm{H}_{9}\right)_{4}, \mathrm{TBT}\right]$, $\mathrm{H}_{2} \mathrm{PtCl}_{6} \cdot 6 \mathrm{H}_{2} \mathrm{O}$, and methyl orange (MO) were purchased from Tianjin Guangfu Fine Chemical Research Institute (China); ethanol, glycerol, and methanol were from Tianjin Yuanli Chemical Co., Ltd; Phenol was from J\&K Chemical; RhB and
Rose Bengal were from Aladdin Industry Corporation; Milli-Q ultrapure water with a resistivity higher than $18.2 \mathrm{M} \Omega \cdot \mathrm{cm}$ was used in all experiments. All the reagents were analytical grade and used as received.

$2 \mathrm{~g}$ TBT was added into a solution of $m(m=0,5,10,20,30) \mathrm{mL}$ glycerol and $(80-m) \mathrm{mL}$ ethanol under a magnetic stirring, and the solution was transferred into a $100 \mathrm{~mL}$ Teflon-lined autoclave and heated at $180^{\circ} \mathrm{C}$ for $24 \mathrm{~h}$. Then, the produced white powders (glycerolates) were collected, washed with absolute ethanol, dried at $60^{\circ} \mathrm{C}$ overnight (labeled as G-m), and finally calcined in air at $470{ }^{\circ} \mathrm{C}$ for $1 \mathrm{~h}$ with a heating rate of $5^{\circ} \mathrm{C} \min ^{-1}$ (labeled as $\mathrm{TiO}_{2}-\mathrm{m}$ ).

Structure characterizations. X-ray diffraction (XRD) patterns were recorded using Panalytical X'Pert Pro X-ray diffractometer equipped with $\mathrm{Cu} \mathrm{K} \alpha$ radiation at $40 \mathrm{kV}$ and $140 \mathrm{~mA}$ at a scanning rate of $5^{\circ} \mathrm{min}^{-1}$. Scanning electron microscopy (SEM) images were observed using a field-emission scanning electron microscope (Hitachi, S-4800). Transmission electron microscopy (TEM) analysis was carried out using a Tecnai $\mathrm{G}^{2} \mathrm{~F}-20$ microscope with a field-emission gun operating at $200 \mathrm{kV}$. X-ray photoelectron spectrum (XPS) analysis was conducted with a PHI$1600 \mathrm{X}$-ray photoelectron spectroscope equipped with $\mathrm{Al} \mathrm{K} \alpha$ radiation, and the binding energy was calibrated by the C1s peak $(284.8 \mathrm{eV})$ of contamination carbon. Specific surface area $\left(S_{\mathrm{BET}}\right)$ was calculated based on $\mathrm{N}_{2}$ adsorption/desorption isotherms recorded on a Micromeritics TriStar 3000 instrument at $77 \mathrm{~K}$, and all the samples were outgassed in a vacuum at $150^{\circ} \mathrm{C}$ for $12 \mathrm{~h}$. UV-vis diffuse reflectance spectra (UV-vis DRS) were obtained from a Shimadzu UV-2600 spectrometer equipped with a $60 \mathrm{~nm}$ diameter integrating sphere using $\mathrm{BaSO}_{4}$ as reference. Time-resolved fluorescence anisotropy decays were recorded on a FL3 system (Horiba Scientific, Edison, NJ) utilizing the time-correlated single photon count. Raman spectra was recorded by a Raman Microscope (DXR Microscope, ThermoFisher, USA), with a 100-mW 532-nm line of Nd:YAG laser as the excitation source. Thermogravitity (TG) analysis was conducted on a TGA Q500 thermogravimeter under an air flow with a rate of $5^{\circ} \mathrm{C} \mathrm{min}^{-1}$. Mott-Schottky (MS) plots were obtained by a capacitance measurement in a standard three-electrode setup (the electrolyte was $0.2 \mathrm{M} \mathrm{Na}_{2} \mathrm{SO}_{4}$ electrolyte; the reference electrode was $\mathrm{Ag} / \mathrm{AgCl}$; and a Pt wire was used as the counter electrode; Autolab potentiostat/galvanostat, Model PGSTAT 302N), where $\mathrm{TiO}_{2}$ powders (after being grinded) spin-coating on F-doped tin oxide (FTO) glass were used as the working electrode. Electrochemical impedance spectroscopy (EIS) measurements were carried out with a sinusoidal ac perturbation of $10 \mathrm{mV}$ applied over the frequency range of $0.01-10^{5} \mathrm{~Hz}$.

X-ray absorption fine structure spectroscopy (XAFS) was performed at the 1W2B beamline of Beijing Synchrotron Radiation Facility. The Ti L-edge XAS spectra were collected on the beamline BL01C1 in NSRRC. Positron annihilation lifetime spectra (PALS) were measured with a fast/slow coincidence ORTEC system with a time resolution of $\sim 201 \mathrm{ps}$ (full width at half-maximum). Fourier transform infrared (FT-IR) spectra were recorded on a BioRad FTS 6000 spectrometer. All samples were mixed with $\mathrm{KBr}$ and pressed into a thin plate for measurement. Solidstate CP/MAS ${ }^{13} \mathrm{C}-\mathrm{NMR}$ spectrum were tested using Infinityplus 300 , Varian, USA.

The concentration of Ti atom (Ti/O ratio) in the bulk of oxide was analyzed by chemical titration method. $0.1 \mathrm{~g}$ of the sample was heated slowly to $110^{\circ} \mathrm{C}$ for $2 \mathrm{~h}$ to evaporate volatile ingredients. Then it was mixed with $4 \mathrm{~g}$ sodium peroxide $\left(\mathrm{Na}_{2} \mathrm{O}_{2}\right)$ and then heated to $800^{\circ} \mathrm{C}$ for $5 \mathrm{~min}$ to remove residual organic materials After that, it was transferred to a $300 \mathrm{~mL}$ beaker, with further $50 \mathrm{~mL}$ water, $30 \mathrm{~mL}$ sulfuric acid $\left(9 \mathrm{~mol} \mathrm{~L}^{-1}\right)$ and $30 \mathrm{~mL}$ hydrochloric acid $\left(12 \mathrm{~mol} \mathrm{~L}^{-1}\right)$ added. The mixture was heated until it was dissolved completely. After cooling, the solution was transferred to a $500 \mathrm{~mL}$ Erlenmeyer flask, and $40 \mathrm{~mL}$ hydrochloric acid $\left(0.6 \mathrm{~mol} \mathrm{~L}^{-1}\right)$ was added. After that, $2 \mathrm{~g}$ of metallic aluminum was added to reduce Ti (IV) ions to Ti (III) ions. The next stage was the titration of this solution with standardized ferric ammonium sulfate solution to the sharp color change from violet to red. Ammonium thiocyanate solution $\left(\mathrm{NH}_{4} \mathrm{SCN}, 300 \mathrm{~g} \mathrm{~L}^{-1}\right)$ was used as the indicator of the end-point. The titration was repeated at least 3 times for each sample, with the experimental error less than $0.5 \%$. The Ti/O ratio on the surface was calculated according to the surface concentration of Ti and O detected by XPS analysis.

Photoelectrochemical (PEC) properties were measured using a CHI660E electrochemical workstation in a three-electrode cell with a $\mathrm{Pt}$ wire as the counter electrode and an $\mathrm{Ag} / \mathrm{AgCl}$ reference electrode. $\mathrm{Na}_{2} \mathrm{SO}_{4}(0.2 \mathrm{M})$ was used as electrolyte solution. The working electrode was prepared by the spin-coating $\mathrm{TiO}_{2}$ slurry on the FTO glass electrode $(1 \mathrm{~cm} \times 1 \mathrm{~cm})$ and heating at $300^{\circ} \mathrm{C}$ for $1 \mathrm{~h}$. Potentials vs. $\mathrm{Ag} / \mathrm{AgCl}$ were converted into potentials $v$ s. reversible hydrogen electrode (RHE) according to the Nernst equation $\left(E_{\mathrm{RHE}}=E_{\mathrm{Ag} / \mathrm{AgCl}}+0.059 \mathrm{pH}+\right.$ $0.196)$. The $J-V$ curves were measured with a scanning rate of $10 \mathrm{mV} \mathrm{s}^{-1}$ under the irradiation of a xenon lamp $(300 \mathrm{~W})$

Photocatalytic tests. Photocatalytic hydrogen production was carried out in a top-irradiation Pyrex reaction vessel connected to closed glass gas system. $10 \mathrm{mg}$ catalyst were dispersed in $100 \mathrm{~mL}$ aqueous solution containing methanol $(30 \mathrm{vol} \%)$ $0.5 \mathrm{wt} \% \mathrm{Pt}$ was introduced as cocatalyst by in situ photodeposition. The temperature of reaction solution was maintained at $0.5^{\circ} \mathrm{C} \pm 0.1{ }^{\circ} \mathrm{C}$ and the light source is a $300 \mathrm{~W}$ high-pressure xenon lamp $\left(100 \mathrm{~mW} \mathrm{~cm}^{-2}\right.$, PLS-SXE 300UV). The produced hydrogen was analyzed using an online gas chromatography (Bruker 450$\mathrm{GC}$, thermal conductive detector, $5 \AA$ molecular sieve column, and $\mathrm{N}_{2}$ as carrier 
gas). For revealing the catalytic reaction pathway difference between n-type $\mathrm{TiO}_{2}-0$ and p-type $\mathrm{TiO}_{2}-10$, the gas products, and liquid products of the photocatalysis are further determined by gas chromatography and liquid chromatography, respectively, and the results indicate that the reaction products are same (with gas products of $\mathrm{CO}_{2}$ and $\mathrm{H}_{2}$, and liquid products of formaldehyde, without formic acid formed since its spontaneous decomposition to $\mathrm{CO}_{2}$ and $\mathrm{H}_{2} \mathrm{O}^{50}$ ) whether n-type or p-type $\mathrm{TiO}_{2}$ is used as photocatalyst (see the details in Supplementary Fig. 34).

Photodegradation of organic pollutants was conducted in an opening quartz chamber $(150 \mathrm{~mL})$ vertically irradiated by a $300 \mathrm{~W}$ high-pressure xenon lamp located on the upper position. The light density was controlled at $100 \mathrm{~mW} \mathrm{~cm}^{-2}$. The irradiation area was ca. $50 \mathrm{~cm}^{2}$. Reaction conditions: temperature, $25 \pm 0.2^{\circ} \mathrm{C}$; $C_{0}(\mathrm{MO})=120 \mu \mathrm{mol} \mathrm{L}^{-1} ; C_{0}(\mathrm{Phenol})=400 \mu \mathrm{mol} \mathrm{L}^{-1} ; C_{0}(\mathrm{RhB})=20 \mu \mathrm{mol} \mathrm{L}-1$; $C_{0}($ Rose Bengal $)=20 \mu \mathrm{mol} \mathrm{L}^{-1}$ and $\mathrm{TiO}_{2}: 0.2 \mathrm{~g} \mathrm{~L}^{-1}$; no acid or alkaline reagents were added. Reaction was conducted by magnetic stirring under atmosphere after stirring for $20 \mathrm{~min}$ in darkness to achieve adsorption equilibrium. Samples were withdrawn, centrifuged, and analyzed using UV-vis spectrometer (UV-2600, Shimadzu Ltd.).

Photocatalytic degradation under magnetic field. Photodegradation of organic pollutants was conducted in a NMR tube (Diameter: $0.5 \mathrm{~cm}$, Length: $18 \mathrm{~cm}$, Content: $2 \mathrm{~mL}$ ) vertically irradiated by a $300 \mathrm{~W}$ high-pressure xenon lamp ( $100 \mathrm{~mW} \mathrm{~cm}^{-2}$, PLS-SXE $300 \mathrm{UV}$ ) located on the upper position, using the vibrating sample magnetometer (LDJ 9600). The irradiation area was $c a .8 \mathrm{~cm}^{2}$. Reaction conditions: temperature, $25 \pm 0.2{ }^{\circ} \mathrm{C} ; C_{0}(\mathrm{MO})=120 \mu \mathrm{mol} \mathrm{L}^{-1} ; C_{0}(\mathrm{RhB})=20 \mu \mathrm{mol}$ $\mathrm{L}^{-1} ; C_{0}$ (Rose Bengal) $=20 \mu \mathrm{mol} \mathrm{L}^{-1}$ and $\mathrm{TiO}_{2}: 2 \mathrm{~g} \mathrm{~L}^{-1}$; no acid or alkaline reagents were added. Samples were withdrawn, centrifuged, and analyzed using UV-vis spectrometer (UV-2600, Shimadzu Ltd.).

\section{Data availability}

The source data underlying Figs. 1-4 and Supplementary Figs. 2-4, 6-8, 11-19, 21-26, $28,29,31$ are provided as a Source Data file.

Received: 31 July 2019; Accepted: 30 December 2019; Published online: 21 January 2020

\section{References}

1. Fujishima, A., Zhang, X. \& Tryk, D. A. $\mathrm{TiO}_{2}$ photocatalysis and related surface phenomena. Surf. Sci. Rep. 63, 515-582 (2008).

2. Chen, X. \& Mao, S. S. Titanium dioxide nanomaterials: synthesis, properties, modifications, and applications. Chem. Rev. 107, 2891-2959 (2007).

3. Crossland, E. J. et al. Mesoporous $\mathrm{TiO}_{2}$ single crystals delivering enhanced mobility and optoelectronic device performance. Nature 495, 215-219 (2013).

4. Chen, X. B., Liu, L., Yu, P. Y. \& Mao, S. S. Increasing solar adsorption for photocatalysis with black hydrogenated titanium dioxide nanocrystals. Science 331, 746-750 (2011).

5. Asahi, R., Morikawa, T., Ohwaki, T., Aoki, K. \& Taga, Y. Visible-light photocatalysis in nitrogen-doped titanium oxides. Science 293, 269-271 (2001).

6. Yang, H. G. et al. Anatase $\mathrm{TiO}_{2}$ single crystals with a large percentage of reactive facets. Nature 453, 638-641 (2008)

7. Liu, G. et al. Titanium dioxide crystals with tailored facets. Chem. Rev. 114, 9559-9612 (2014).

8. Zhang, Y.-C., Afzal, N., Pan, L., Zhang, X. \& Zou, J.-J. Structure-activity relationship of defective metal-based photocatalysts for water splitting: experimental and theoretical perspectives. Adv. Sci. 6, 1900053 (2019).

9. Pan, L. et al. Constructing $\mathrm{TiO}_{2}$ p-n homojunction for photoelectrochemical and photocatalytic hydrogen generation. Nano Energy 28, 296-303 (2016).

10. Suntivich, J., May, K. J., Gasteiger, H. A., Goodenough, J. B. \& Shao-Horn, Y. A perovskite oxide optimized for oxygen evolution catalysis from molecular orbital principles. Science 334, 1383-1385 (2011).

11. Chen, $\mathrm{S}$. et al. Delocalized spin states in $2 \mathrm{D}$ atomic layers realizing enhanced electrocatalytic oxygen evolution. Adv. Mater. 29, 1701687 (2017).

12. Zhou, S. et al. Engineering electrocatalytic activity in nanosized perovskite cobaltite through surface spin-state transition. Nat. Commun. 7, 11510 (2016).

13. Zhang, R. et al. Engineering cobalt defects in cobalt oxide for highly efficient electrocatalytic oxygen evolution. ACS Catal. 8, 3803-3811 (2018).

14. Gracia, J., Sharpe, R. \& Munarriz, J. Principles determining the activity of magnetic oxides for electron transfer reactions. J. Catal. 361, 331-338 (2018).

15. Liu, Y. et al. Ultrathin $\mathrm{Co}_{3} \mathrm{~S}_{4}$ nanosheets that synergistically engineer spin states and exposed polyhedra that promote water oxidation under neutral conditions. Angew. Chem. Int. Ed. 54, 11231-11235 (2015).

16. Sun, S. et al. Boosting oxygen evolution kinetics by Mn-N-C motifs with tunable spin state for highly efficient solar-driven water splitting. Adv. Energy Mater. 9, 1901505 (2019).
17. Mtangi, W. et al. Control of electrons' spin eliminates hydrogen peroxide formation during water splitting. J. Am. Chem. Soc. 139, 2794-2798 (2017).

18. Mtangi, W., Kiran, V., Fontanesi, C. \& Naaman, R. Role of the electron spin polarization in water splitting. J. Phys. Chem. Lett. 6, 4916-4922 (2015).

19. Zhang, W., Banerjee-Ghosh, K., Tassinari, F. \& Naaman, R. Enhanced electrochemical water splitting with chiral molecule-coated $\mathrm{Fe}_{3} \mathrm{O}_{4}$ nanoparticles. ACS Energy Lett. 3, 2308-2313 (2018).

20. Ghosh, K. B. et al. Controlling chemical selectivity in electrocatalysis with chiral CuO-coated electrodes. J. Phys. Chem. C. 123, 3024-3031 (2019).

21. Abendroth, J. M. et al. Spin selectivity in photoinduced charge-transfer mediated by chiral molecules. ACS Nano 13, 4928-4946 (2019).

22. Naaman, R., Paltiel, Y. \& Waldeck, D. H. Chiral molecules and the electron spin. Nat. Rev. Chem. 3, 250-260 (2019).

23. Michaeli, K., Kantor-Uriel, N., Naaman, R. \& Waldeck, D. H. The electron's spin and molecular chirality-how are they related and how do they affect life processes? Chem. Soc. Rev. 45, 6478-6487 (2016).

24. Garcés-Pineda, F. A., Blasco-Ahicart, M., Nieto-Castro, D., López, N. \& Galán-Mascarós, J. R. Direct magnetic enhancement of electrocatalytic water oxidation in alkaline media. Nat. Energy 4, 519-525 (2019).

25. Wang, S. et al. Titanium-defected undoped anatase $\mathrm{TiO}_{2}$ with p-type conductivity, room-temperature ferromagnetism, and remarkable photocatalytic performance. J. Am. Chem. Soc. 137, 2975-2983 (2015).

26. Pan, L. et al. Undoped $\mathrm{ZnO}$ abundant with metal vacancies. Nano Energy 9 , 71-79 (2014)

27. Wu, S.-M. et al. Homojunction of oxygen and titanium vacancies and its interfacial n-p effect. Adv. Mater. 30, 1802173 (2018).

28. Das, J., Freitas, F. S., Evans, I. R., Nogueira, A. F. \& Khushalani, D. A facile nonaqueous route for fabricating titania nanorods and their viability in quasisolid-state dye-sensitized solar cells. J. Mater. Chem. 20, 4425-4431 (2010).

29. Chen, Y. et al. Solvothermal synthesis, characterization, and formation mechanism of a single-layer anatase $\mathrm{TiO}_{2}$ nanosheet with a porous structure. Eur. J. Inorg. Chem. 2011, 754-760 (2011).

30. Thushara, K. S. et al. $\mathrm{MgCl}_{2} \cdot 4\left(\mathrm{CH}_{3}\right)_{2} \mathrm{CHOH}$ : A new molecular adduct and super active polymerization catalyst support. J. Phys. Chem. C. 113, 8556-8559 (2009).

31. Pei, D. N. et al. Defective titanium dioxide single crystals exposed by high-energy $\{001\}$ facets for efficient oxygen reduction. Nat. Commun. 6, 8696 (2015).

32. Jiang, $\mathrm{X}$. et al. Characterization of oxygen vacancy associates within hydrogenated $\mathrm{TiO}_{2}$ : a positron annihilation study. J. Phys. Chem. C. 116, 22619-22624 (2012).

33. Li, X., Guo, Z. \& He, T. The doping mechanism of $\mathrm{Cr}$ into $\mathrm{TiO}_{2}$ and its influence on the photocatalytic performance. Phys. Chem. Chem. Phys. 15, 20037-20045 (2013)

34. Kong, M. et al. Tuning the relative concentration ratio of bulk defects to surface defects in $\mathrm{TiO}_{2}$ nanocrystals leads to high photocatalytic efficiency. J. Am. Chem. Soc. 133, 16414-16417 (2011).

35. Angelomé, P. C. et al. Mesoporous anatase $\mathrm{TiO}_{2}$ films: use of Ti K XANES for the quantification of the nanocrystalline character and substrate effects in the photocatalysis behavior. J. Phys. Chem. C. 111, 10886-10893 (2007).

36. Cao, J., Zhang, Y., Liu, L. \& Ye, J. A p-type Cr-doped $\mathrm{TiO}_{2}$ photo-electrode for photo-reduction. Chem. Commun. 49, 3440-3442 (2013).

37. Tian, B. et al. Supported black phosphorus nanosheets as hydrogen-evolving photocatalyst achieving $5.4 \%$ energy conversion efficiency at 353 K. Nat. Commun. 9, 1397 (2018).

38. Na-Phattalung, S. et al. First-principles study of native defects in anatase $\mathrm{TiO}_{2}$ Phys. Rev. B 73, 125205 (2006)

39. Ohtomo, A., Muller, D. A., Grazul, J. L. \& Hwang, H. Y. Artificial chargemodulationin atomic-scale perovskite titanate superlattices. Nature 419, 378-380 (2002).

40. Jiang, B. et al. Enhanced photocatalytic activity and electron transfer mechanisms of graphene/TiO ${ }_{2}$ with exposed $\{001\}$ facets. J. Phys. Chem. C. 115, 23718-23725 (2011).

41. Caffrey, N. M., Ferriani, P., Marocchi, S. \& Heinze, S. Atomic-scale inversion of spin polarization at an organic-antiferromagnetic interface. Phys. Rev. B 88, 155403 (2013)

42. Zhang, Z. \& Yates, J. T. Band bending in semiconductors: chemical and physical consequences at surfaces and interfaces. Chem. Rev. 112, 5520-5551 (2012).

43. Schwabl \& Franz. Quantum Mechanics (Springer-Verlag, 1991).

44. Baibich, M. N. et al. Giant magnetoresistance of (001) Fe/(001) Cr magnetic superlattices. Phys. Rev. Lett. 61, 2472-2475 (1988).

45. Li, J. et al. Enhanced photocatalytic performance through magnetic field boosting carrier transport. ACS Nano 12, 3351-3359 (2018).

46. Jiao, Y., Sharpe, R., Lim, T., Niemantsverdriet, J. W. H. \& Gracia, J. Photosystem II acts as a spin-controlled electron gate during oxygen formation and evolution. J. Am. Chem. Soc. 139, 16604-16608 (2017).

47. Peng, H., Li, J., Li, S.-S. \& Xia, J.-B. Possible origin of ferromagnetism in undoped anatase $\mathrm{TiO}_{2}$. Phys. Rev. B 79, 092411 (2009). 
48. Kresse, G. \& Furthmuller, J. Efficient iterative schemes for ab initio totalenergy calculations using a plane-wave basis set. Phys. Rev. B 54, 11169-11186 (1996).

49. Meng, Q. et al. Understanding electronic and optical properties of anatase $\mathrm{TiO}_{2}$ photocatalysts co-doped with nitrogen and transition metals. Phys. Chem. Chem. Phys. 15, 9549-9561 (2013).

50. Schneider, J. \& Bahnemann, D. W. Undesired role of sacrificial reagents in photocatalysis. J. Phys. Chem. Lett. 4, 3479-3483 (2013).

\section{Acknowledgements}

The authors appreciate the support from the National Natural Science Foundation of China (21978200, 21676193, 51661145026) and the Scientific Research Projects of the Ministry of Education of China (6141A02033522). The authors also appreciate the help of XAFS analysis from Beijing Synchrotron Radiation, Institute of High Energy Physics, Chinese Academy of Sciences, Beijing, China.

\section{Author contributions}

Z.J. conceived the idea and designed this study. P.L., A.M. and H.C. contributed equally to this work, and synthesized the samples and conducted characterizations. M.W., Y.L. and Z.R. conducted computations. P.L., H.C., A.M., L.X. and W.S. performed photocatalytic reactions. P.L., Z.J. and J.Z analyzed the XAS tests. Z.J., P.L., H.C., A.M., M.W. and Z.X. wrote the paper. All authors discussed the results and edited the paper.

\section{Competing interests}

The authors declare no competing interests.

\section{Additional information}

Supplementary information is available for this paper at https://doi.org/10.1038/s41467020-14333-w.

Correspondence and requests for materials should be addressed to J.-J.Z. or W.M.

Peer review information Nature Communication thanks Detlef Bahnemann, Nuala Caffrey, and Ron Naaman for their contributions to the peer review of this work.

Reprints and permission information is available at http://www.nature.com/reprints

Publisher's note Springer Nature remains neutral with regard to jurisdictional claims in published maps and institutional affiliations.

(c) (i) Open Access This article is licensed under a Creative Commons Attribution 4.0 International License, which permits use, sharing, adaptation, distribution and reproduction in any medium or format, as long as you give appropriate credit to the original author(s) and the source, provide a link to the Creative Commons license, and indicate if changes were made. The images or other third party material in this article are included in the article's Creative Commons license, unless indicated otherwise in a credit line to the material. If material is not included in the article's Creative Commons license and your intended use is not permitted by statutory regulation or exceeds the permitted use, you will need to obtain permission directly from the copyright holder. To view a copy of this license, visit http://creativecommons.org/ licenses/by/4.0/.

(C) The Author(s) 2020 